Revue

de I'histoire des religions

Revue de l'histoire des religions

$4 \mid 2021$

La magie entre Antiquité et Moyen Âge : traditions, innovations, autorités

\title{
La magie entre Antiquité et Moyen Âge : traditions, innovations, autorités
}

Avant-propos

Thomas Galoppin

\section{CpenEdition}

\section{Journals}

Édition électronique

URL : https://journals.openedition.org/rhr/11464

DOI : $10.4000 /$ rhr. 11464

ISSN : 2105-2573

Éditeur

Armand Colin

\section{Édition imprimée}

Date de publication : 1 décembre 2021

Pagination : 603-616

ISBN : 978-2-200-93378-4

ISSN : 0035-1423

Référence électronique

Thomas Galoppin, "La magie entre Antiquité et Moyen Âge : traditions, innovations, autorités », Revue de l'histoire des religions [En ligne], 4 | 2021, mis en ligne le 01 décembre 2021, consulté le 06 janvier 2022. URL : http://journals.openedition.org/rhr/11464 ; DOI : https://doi.org/10.4000/rhr.11464 


\section{La magie entre Antiquité et Moyen Âge : traditions, innovations, autorités Avant-propos}

« Je fuis l'innovation en toutes choses, je puis le dire, et spécialement en ce qui concerne les dieux »

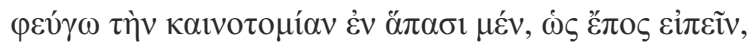

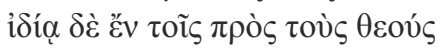
Julien, Lettres, 89 [453b].

Le GIS (Groupement d'intérêt scientifique) «Religions - Pratiques, textes, pouvoirs » avait invité les chercheurs, dans le cadre d'un congrès sur «La Tradition» auquel les contributeurs de ce dossier ont participé les 8-10 octobre 2019, à penser l'épaisseur temporelle des «faits religieux » telle qu'elle est rendue sensible dans la transmission de savoirs et de rites ${ }^{1}$. Toute tradition, on le sait, se déploie dans un espace-temps particulier. Elle n'est donc pas imperméable aux dynamiques sociales et historiques. Les savoirs religieux, comme n'importe quels savoirs, n'ont rien de figé, même pour des systèmes fondés sur un texte «révélé »; ils sont nourris de transferts culturels qui passent par toutes sortes de bricolages lors de processus d'appropriation. Pareillement, les rites ne cessent de s'adapter, d'innover parfois, avec des matériaux nouveaux. De

1. Programme accessible sur le site du GIS Religions (http://gis-religions.fr/ actualites/2019-congres) comprenant en particulier deux sessions «Magie, rites, savoirs : une tradition de l'innovation? » organisées par Nicole Belayche (que je remercie pour sa présence aux diverses étapes de cette publication) et moi-même. 
façon finalement guère provocante depuis de nombreux travaux sur la dynamique des rituels ${ }^{2}$, on peut dire que plus une société se revendique comme construite sur la tradition - par exemple, la Rome antique qui se réfère en permanence au mos maiorum (la tradition des ancêtres) -, plus les observateurs que nous sommes sont invités à rechercher les points d'innovation ${ }^{3}$, et donc à en penser les processus et les modalités. Pour les pratiques et les savoirs magiques, tradition et innovation sont des lignes de force dont l'articulation préside à leur composition : ces rites s'ancrent dans une ou plusieurs tradition(s) et sont dans le même temps le lieu d'innovations constantes - dont ce dossier éclaire certaines rationalités - parce que leurs praticiens n'appartiennent pas à des catégories institutionnelles ou institutionnalisées. L'établissement de leur autorité représente un défi auquel répondre en tramant la nouveauté sur une chaîne de tradition.

\section{Pratiques magiques et nOtion de « MAgie »}

Ce qu'on appelle aujourd'hui « magie » en histoire des religions rassemble aussi bien des pratiques de guérison que, à l'opposé, des pratiques de malédiction, des techniques visant à assurer une réussite économique ou sociale ou à procurer des moyens de « séduction »-pour le dire avec euphémisme. Ces finalités liées à l'ignorance du futur et aux risques de la vie sont poursuivies par des moyens rituels. Lorsque ces derniers échappent à un cadre institutionnel ou perdent l'aval des autorités religieuses, politiques, voire intellectuelles, ces « autorités » emploient des qualifications propres au champ lexical de la «magie» pour les désigner. Cela ne signifie pas que les rituels incriminés fonctionnent sur un mode différent du ritualisme "patenté », car ils continuent d'appartenir aux systèmes politico-religieux qui s'en défient - tout en $\mathrm{y}$

2. Par exemple : Jens Kreinath, Constance Hartung, Annette Deschner éd., The Dynamics of Changing Rituals: The Transformation of Religious Rituals within their Social and Cultural Context, New York/Oxford, P. Lang, 2004 et Angelos Chaniotis éd., Ritual Dynamics in the Ancient Mediterranean: Agency, Emotion, Gender, Representation, Stuttgart, Franz Steiner, 2011.

3. Les documents le disent sous des dehors de «fidéisme», par exemple pour la Grèce le règlement des mystères d'Andanie (Laura Gawlinski, The Sacred Law of Andania: A New Text with Commentary, Berlin, De Gruyter, 2012) et pour Rome les ludi saeculares (Zosime, II, 6). 
recourant parfois ${ }^{4}$ - et, pour le dire autrement, ils s'attachent à des (ou à leurs) traditions religieuses tout en disposant d'une plus grande marge de manœuvre par rapport à elles.

Le mot de «magie» vient du grec ancien $\mu \alpha \gamma \varepsilon i ́ \alpha$ passé par le latin magia. En grec, le magos ( «mage»), calque probable du vieux perse maguš, désignait prioritairement un type d'individu, prêtre dans l'Empire achéménide ${ }^{5}$. En utilisant ce terme avec tout un lexique du lien et de la fascination, aux côtés de la goêteia (qui recouvre des capacités d'enchantement) ou des pharmaka, substances puissantes (remèdes ou poisons), les lettrés de la Grèce du $\mathrm{v}^{\mathrm{e}}$ siècle avant notre ère (philosophes, médecins, auteurs tragiques et ethnographes) ont voulu singulariser divers experts rituels qui échappaient à une «norme» religieuse qu'eux-mêmes proposaient ${ }^{6}$. En nommant ces praticiens d'un mot qui en connotait le caractère étranger, voire ennemi, ces autorités de la pensée entendaient les affubler d'une altérité culturelle radicale. Pourtant, catégoriser à part les ligatures, les incantations, les amulettes, dans la Grèce de Socrate, est un mouvement historique en soi, appelé à une lente maturation au cours de l'Antiquité dans la mesure où le degré d'admission de ces pratiques, qui ne se développent pas sans continuité avec les traditions rituelles approuvées, peut changer selon les auteurs, les acteurs, les contextes ${ }^{7}$.

Le latin de la Rome républicaine n'avait pas de mot pour dire la «magie ${ }^{8}$ et lorsque Cicéron le premier emploie le terme magus,

4. Voir Libanios, Discours, 41 (Magi repulsa) Inc. (Foerster 7, 367), lors d'une épidémie à Antioche.

5. Albert De Jong, Traditions of the Magi: Zoroastrianism in Greek and Latin Literature, Leyde, Brill, 1997.

6. Fritz Graf, La magie dans l'Antiquité gréco-romaine. Idéologie et pratique, Paris, Les Belles Lettres, 1994, p. 31-45; «Excluding the Charming: The Development of the Greek Concept of Magic », Ancient Magic and Ritual Power, éd. Marvin Meyer, Paul Mirecki, Leyde, Brill, 1995, p. 29-42 ; Jan N. Bremmer, "The Birth of the Term "Magic" ", The Metamorphosis of Magic from Late Antiquity to the Early Modern Period, éd. Jan N. Bremmer, Jan R. Veenstra, Louvain, Peeters, 2002, p. 1-11 (révisé dans Zeitschrift für Papyrologie und Epigraphik, t. 126, 1999, p. 1-12) ; Cléo M. Carastro, La cité des mages. Penser la magie en Grèce ancienne, Grenoble, Jérôme Millon, 2006.

7. Voir les contributions dans David Frankfurter éd., Guide to the Study of Ancient Magic, Leyde, Brill, 2019, partie 2, «Cultural Constructions of Ambiguous, Unsanctioned, or Illigetimate Ritual », p. 27-276.

8. James B. Rives, «Magus and its cognates in Classical Latin», Magical Practice in the Latin West. Papers from the International Conference held at the University of Zaragoza, 30 Sept.-1 Oct. 2005, éd. Richard Gordon, Francisco Marco Simón, Leyde, Brill, 2010, p. 53-77. 
au $\mathrm{I}^{\mathrm{er}}$ siècle avant notre ère, c'est encore pour désigner les prêtres perses ${ }^{9}$. Sous l'Empire en revanche, les magi sont décrits comme les détenteurs d'un savoir d'origine orientale, appliqué aux rites religieux et à la médecine, mais de façon dangereuse parce que mensongère. Au $\mathrm{I}^{\mathrm{er}}$ siècle de notre ère, Pline l'Ancien modèle une histoire de l'ars magica en tant que savoir perse qui, s'insinuant dans le monde depuis les Guerres médiques à travers les écrits de mages, de grammairiens ou de pseudo-philosophes et médecins grecs, prend les atours de la médecine, des traditions rituelles et de l'astrologie ${ }^{10}$. À la même époque, ce sont bien les astrologues et les devins consultés en privé que les cercles du pouvoir impérial désignent du nom de «mages » et tentent de tenir à distance des jeux politiques ${ }^{11}$. Le terme de magia n'apparait qu'au II $^{\mathrm{e}}$ siècle de notre ère, dans la défense du philosophe et rhéteur africain Apulée qui récuse l'accusation de pratiques déviantes pour charger le terme d'une connotation positive, dans la mouvance des « sagesses barbares $»^{12}$.

La notion de «magie» est un objet historique en soi dont on saisit les enjeux en observant comment et par qui elle est progressivement construite de façon à servir de miroir à l'établissement d'une norme religieuse ${ }^{13}$. En parallèle, faire

9. Cicéron, De la divination, I, 46 ; Lois, II, 26.

10. Pline l'Ancien, Histoire naturelle, XXX, 1.

11. Yann Rivière, "L'exil des mages et des sages. Un empire sans philosophes ? ( $\mathrm{I}^{\mathrm{er}}$ siècle ap. J.-C.) », Philosophari. Usages romains des savoirs grecs sous la République et l'Empire, dir. Pierre Vesperini, Paris, Classiques Garnier, 2017, p. 265-352. Pour une vue synthétique de ces dynamiques propres au monde romain, je me permets de renvoyer à Thomas Galoppin, "Magie", malédictions et pouvoir dans le monde romain de la deuxième guerre punique à la fin des Sévères », L'autel et la toge. Religion et pouvoir dans le monde romain de la deuxième guerre punique à la fin des Sévères, dir. Nicole Belayche, Sylvia Estienne, Rennes, Presses universitaires de Rennes, 2020, p. 253-273.

12. Apulée, Apologie, 25-26. Arnaldo Momigliano, Sagesses barbares: les limites de l'hellénisation, Paris, Maspéro, 1979 (trad. fr. de Alien Wisdom. The Limits of Hellenization, Cambridge, Cambridge University Press, 1975).

13. Radcliffe G. Edmonds III, Drawing Down the Moon: Magic in the Ancient Greco-Roman World, Princeton/Oxford, Princeton University Press, 2019. Voir Pierre Brulé, «En guise de prélude... », La norme en matière religieuse en Grèce ancienne. Actes du XII colloque international du CIERGA (Rennes, septembre 2007), dir. Pierre Brulé, Liège, Presses universitaires de Liège, 2009, p. 7-11; Sylvia Estienne, Valentino Gasparini, Anne-Françoise Jaccottet, Jörg Rüpke, « La religion romaine : une fabrique de la norme ?», La norme sous la République et le Haut-Empire romains. Élaboration, diffusion et contournements, éd. Tanja Itgenshorst, Philippe Le Doze, Bordeaux, Ausonius, 2017, p. 201-216. 
l'histoire des pratiques qui ont pu être considérées comme « magiques » conduit à explorer des jeux d'adaptation en suivant la transmission des savoirs selon des canaux différents, au gré des dynamiques sociales et politiques et des rencontres culturelles dans un monde méditerranéen ouvert ${ }^{14}$. Dans le cadre des savoirs magiques, on observe alors des transferts, des adaptations, des hybridations culturelles, tout particulièrement observés dans les documents de l'Antiquité tardive qui montrent la construction d'un ritualisme profondément multiculturel. Un tel ritualisme se démarque en ce qu'il puise à différentes traditions - qu'il s'agisse de la religion grecque, égyptienne, des religions ouest-sémitiques ou encore des religions monothéistes existantes: croisement des cultures, un tel ritualisme est jugé «magique » précisément parce qu'il ne se limite pas aux normes établies par une tradition culturelle donnée, qui se prétend généralement «indigène » ou «ethnique $»^{15}$.

Or, de la fin de l'Antiquité au haut Moyen Âge, une «tradition commune de la magie $»^{16}$ se dessine et prend des caractéristiques propres. La christianisation des pouvoirs s'accompagne d'une définition doctrinale de la notion de «magie», tandis que les pratiques elles-mêmes se transforment: le haut Moyen Âge, en Occident comme en Orient, opère une adaptation des rituels antiques, christianisés ici, islamisés là, tandis que de nouvelles procédures apparaissent. Le corpus d'une magie «savante» ou «naturelle» s'étoffe et constitue peu à peu une catégorie autonome de «magie » en tant que champ disciplinaire, dans les sphères savantes, religieuses et princières de l'Europe des $\mathrm{XII}^{\mathrm{e}}$ et $\mathrm{XIII}^{\mathrm{e}}$ siècles $^{17}$.

14. Véronique Dasen, Jean-Michel Spieser éd., Les savoirs magiques et leur transmission, Florence, Sismel - Edizioni del Galluzzo, 2014. Voir aussi Attilio Mastrocinque, Joseph E. Sanzo, Marianna Scapini éd., Ancient Magic: Then and Now, Stuttgart, Franz Steiner, 2020.

15. Le cas romain est le plus exemplaire, malgré un « vieux Romain » comme Caton qui recommandait des rituels curatifs de conception « magique », Caton, $D e$ l'agriculture, 160.

16. L'expression est de Richard Kieckhefer, Magic in the Middle Ages, Cambridge, [1989] 2000, chap. 4 (« The common tradition of Medieval magic »), p. $56-94$.

17. Nicolas Weill-Parot, Les «Images astrologiques » au Moyen Âge et à la Renaissance. Spéculations intellectuelles et pratiques magiques (XII ${ }^{e}-X V^{e}$ siècles), Paris, Honoré Champion, 2002 ; Jean-Patrice Boudet, Entre science et nigromance. 
Les contributions de ce dossier se concentrent sur l'Antiquité tardive et le Moyen Âge, et interrogent des documents «magiques» qui appartiennent soit à un monde polythéiste, soit à un monde chrétien. Florian Audureau montre à quel point l'historiographie sur la «magie» recourt elle-même à des images traditionnelles, comme celle du magicien astrologue, pour expliquer des phénomènes comme l'usage performatif des voyelles grecques. Toutefois, c'est précisément à un processus d'innovation qu'appartient cet usage, lorsqu'il participe à la construction multiculturelle de rituels dans l'Égypte grécoromaine. Un document en particulier permet d'aborder cette construction du savoir, le papyrus grec magique XIII, que Thomas Galoppin convoque également mais dans une autre perspective, pour explorer la construction d'un design, celui d'images rituelles qui composent une représentation de la divinité en dépassant la différence entre écriture et image. Une telle innovation peut être comprise dans l'Antiquité tardive comme une réception parmi d'autres du modèle des hiéroglyphes égyptiens et elle revendique de la sorte une tradition égyptienne plus ancienne. L'innovation se présente alors comme la fabrique d'une autorité traditionnelle.

\section{TRADITION, INNOVATION ET AUTORITÉ}

Sans appartenir de plein droit à une tradition religieuse bien identifiée, les pratiques «magiques » restent dans la continuité des différentes traditions auxquelles elles puisent. Elles passent par des canaux de transmission divers, en grande partie oraux à côté de recettes écrites, et c'est dans cette dynamique de la transmission que l'on mesure à la fois l'originalité et la permanence d'un savoir magique. Une bonne part des rites «magiques » relève d'un savoir partagé, diffus, qui n'est pourtant pas l'apanage de «magiciens » professionnels ; certaines techniques plus complexes, en revanche, témoignent d'une expertise individuelle. Un des éléments clés pour analyser les liens entre «magie» et tradition est l'acteur principal de cette dynamique de transmission: l'expert rituel. 
Le modèle gréco-romain du «mage » en tant que détenteur d'un savoir spécifique, croisant des savoirs naturalistes et des rites religieux dans la sphère privée, recouvre une réalité : des hommes et des femmes qui offrent des services voisins de ceux des prêtres ou des médecins, mais sans l'accréditation d'une autorité officielle. En termes d'élaboration de ce modèle, l'Esquisse générale d'une théorie de la magie de Marcel Mauss et Henri Hubert, parue en 1903, fut le fruit d'une collaboration entre un sociologue et un latiniste. Les catégories antiques ont donc informé les catégories anthropologiques modernes qui mettent en relief les particularités de l'individu «magicien»: seul/isolé, hors cadre institutionnel, pratiquant dans le secret. C'est autour des experts rituels que se cristallisent les enjeux portant sur les rites et les savoirs, puisqu'ils en assurent la transmission ou les transformations par le biais de leurs pratiques. C'est d'ailleurs à une étude de la sociologie des experts indépendants du rituel at the temple gates qu'invite une approche comme celle qu'a menée Heidi Wendt pour les deux premiers siècles de l'Empire romain ${ }^{18}$. Toutefois, la documentation de première main laissée par ces agents rituels est la plupart du temps silencieuse sur leur identité. On peut néanmoins mesurer l'autonomie dont ils bénéficient à travers les adaptations, voire l'invention, qui font le sel de ces pratiques. En dépassant les problèmes d'une définition relative de la notion de magie, observer la dynamique du savoir qui résulte d'une articulation entre tradition et innovation est ainsi l'un des meilleurs moyens de connaître ces agents et les enjeux culturels auxquels ils faisaient face.

Une tradition est un ensemble de pratiques normées et ritualisées qui perpétuent, et donc transmettent, certaines conventions en les légitimant par référence au passé. Cette référence caractérise le processus d'invention des traditions selon le modèle d'Eric Hobsbawm $^{19}$. Dans ce cadre théorique qui fait consensus, les traditions se fabriquent de façon dynamique en accompagnant des transformations sociales. En ce sens, une tradition participe des pratiques qui forgent une identité collective. Or, la notion même de

18. Heidi Wendt, At the Temple Gates: The Religion of Freelance Experts in the Roman Empire, New York/Oxford, Oxford University Press, 2016.

19. Eric Hobsbawm, «Introduction: Inventing Traditions », The Invention of Tradition, éd. Eric Hobsbawm et Terence Ranger, Cambridge, Cambridge University Press, 1983, p. 1-14. 
« magie » est une manière de construire une altérité généralement individualisée, celle d'experts rituels qui ne forment pas, a priori, de communauté, ni ne s'inscrivent dans une institution. Mais ces experts sont les agents d'une transmission au sens étymologique de tradere, d'une traditio. L'idée même d'une transmission, dans le domaine des savoirs, peut faire débat: dans le monde grécoromain, par exemple, le savoir comme sagesse (sophia) est objet d'émulation plus que de transmission, à la différence des savoirs techniques (technai, artes) ${ }^{20}$. Or, l'ars magica est présentée comme une compétence technique transmise et acquise par les livres, et ce sont des textes qui nous donnent à voir la majeure partie des pratiques. Le papyrus de Derveni, au $\mathrm{IV}^{\mathrm{e}}$ siècle avant notre ère, évoque déjà la figure d'experts dans la technê du rituel ${ }^{21}$. Est-ce précisément cette transmission d'un savoir rituel, technique, qui construit une tradition à part ?

Les rituels groupés dans la catégorie de «magie », laquelle tend à l'assemblage hétéroclite, comprennent tout autant des pratiques divinatoires qui peuvent aller jusqu'à la recherche d'une révélation divine. L'importance de cette dernière en vue de fonder la légitimité de ce qui deviendra une «tradition », parce qu'elle lui donne une autorité22 ${ }^{22}$ explique la récurrence de chaînes de révélation depuis l'Antiquité païenne jusqu' aux ésotérismes de la Renaissance ou de l'époque contemporaine. Dans l'Antiquité tardive et le Moyen Âge déjà, la réception des figures d'autorité comme Hermès, Orphée, Pythagore ou Démocrite, mais aussi Moïse ou Apollonios de Tyane, opère comme une ancre pour amarrer le savoir technique à une tradition rituelle ou philosophique. C'est ainsi que dans le monde musulman médiéval l'héritage antique véhicule avec lui de grands noms ${ }^{23}$. Les noms des figures de la révélation ou des

20. Pierre Vesperini, «La culture antique était-elle une "culture de la transmission" ? Façons grecques et façons romaines de faire passer les savoirs », ASDIWAL. Revue genevoise d'anthropologie et d'histoire des religions, t. 12, 2017, p. 113-134.

21. P. Derveni, $\mathrm{XX}, 4$.

22. Voir l'introduction de Gregory P. Grieve et Richard Weiss, «Illuminating the Half-Life of Tradition: Legitimation, Agency, and Counter-Hegemonies ", Historicizing "Tradition" in the Study of Religion, éd. Steven Engler, Gregory P. Grieve, Berlin, De Gruyter, 2005, p. 1-15.

23. Jean-Charles Coulon, La Magie en terre d'islam au Moyen Âge, Paris, Éditions du Comité des travaux historiques et scientifiques, 2017, p. 65-104. 
fondateurs de la tradition ont une efficacité24 : ils autorisent le savoir et, dès lors, participent à la construction d'une identité qui se veut traditionnelle et donc partagée. L'expert rituel ne s'isole pas : il s'inscrit dans une chaîne de transmission. Dans sa contribution, Korshi Dosoo relit un passage du papyrus grec magique IV et propose d'y retrouver la référence à une autorité mythique : Aiétès, fils du Soleil et père de Médée. La transmission du rituel se trouve ainsi renforcée d'une figure d'autorité. K. Dosoo prolonge ensuite l'enquête sur le terrain chrétien, copte, un contexte où le savoir «magique» s'inscrit pleinement dans la continuité des rituels plus anciens qu'il acclimate au christianisme. La continuité peut aussi se mesurer par rapport à la liturgie copte dans la mesure où l'autorité des textes révélés fonde l'efficience de l'historiola. Le rite «magique» adapte des formes rituelles reconnues à des situations ponctuelles, dans lesquelles il réactualise la puissance de figures reliées au surhumain, comme Marie.

Le recours à la tradition est indissociable des innovations que les experts rituels mettent en œuvre pour adapter leurs pratiques à des contextes mouvants. À titre de comparaison, nous pouvons renvoyer à la manière dont les chamanes de Sibérie (re)construisent une tradition qui favorise dans le même temps une certaine liberté d'innovation, dans un contexte de métamorphoses culturelles et sociales ouvert aux emprunts de tous ordres ${ }^{25}$. L'innovation s'accompagne d'un processus de réflexivité sur les pratiques, ce qui peut être une mise en perspective de l'action rituelle en tant qu'action efficiente ${ }^{26}$. La réflexivité du rituel peut notamment passer par des dispositifs ou une esthétique qui donnent à voir ce «plus » qui est conféré, par le rituel, à l'agent qui y participe : c'est notamment le cas dans les «traditions inventées » de l'ésotérisme ou du néopaganisme contemporains - traditions

24. Anna Van den Kerchove, «Poimandrès, figure d'autorité dans la tradition hermétique », Revue de l'histoire des religions, t. 231, fasc. 1, 2014, p. 27-46.

25. Ksenia Pimenova, «Traditions et emprunts dans un miroir chamanique. Réflexions autour d'un nouveau rituel touva", Retours aux rituels, Ethnographiques.org, 33, 2016 (en ligne : https://www.ethnographiques.org/2016/ Pimenova) ; «Ressources et limites du bricolage rituel. Influences du bouddhisme et de l'ésotérisme dans le chamanisme contemporain en Altaï et à Touva », Transferts culturels en Sibérie de l'Altaï à la Iakoutie, dir. Pavel Alekseev, Ekaterina Dmitrieva, Michel Espagne, Paris, Demopolis, 2018, p. 259-283.

26. Guillaume Rozenberg, "Magie du rituel, démon de la réflexivité », L'Homme, t. 198-199, 2011, p. 277-299. 
friandes de «magie $»^{27}$. Lorsque ce sont des textes ou des images qui construisent, transmettent, autorisent le rituel, l'innovation s'empare des habits d'une tradition, adapte les emprunts ou les transferts qu'elle opère, en utilisant les ressources du discours, des stratégies d'énonciation, des formes visuelles. Ces bricolages sont inhérents à toutes les traditions rituelles ou mythographiques ${ }^{28}$. Mais qu'en est-il lorsqu'il faut "bricoler» au sein d'un savoir suspect, voire réprouvé par les autorités politiques ou religieuses? Comment établir un contact avec la divinité dans l'Égypte tardive, à l'extérieur des sanctuaires et dans un contexte où les autorités romaines, puis chrétiennes, se méfient des divinations privées? Comment négocier, au Moyen Âge, avec le dogme dans un monde où le savoir religieux s'institutionnalise?

Quittant l'Orient grec et copte pour l'occident médiéval latin, c'est en s'attachant au parcours universitaire que l'Ars notoria propose un ritualisme complexe, et en même temps réprouvé par les théologiens. Julien Véronèse montre combien les variations dans l'écriture de ces savoirs magiques, en marge des milieux religieux et savants qui les condamnent, s'adaptent : la réécriture a pour but d'accroître l'efficacité des rites, ou bien de simplifier la tradition, voire de christianiser des rites demeurés suspects.

\section{OUVRIR LA TRADITION}

Un ouvrage de l'Antiquité tardive, composé en grec peut-être au $\mathrm{IV}^{\mathrm{e}}$ siècle à partir de données antérieures, illustre les dynamiques à l'enquête dans ce dossier. L'ouvrage est connu sous le titre de Cyranides et réputé comme un ouvrage de «magie», sans que la question ait jamais été véritablement examinée. Le texte est établi, difficilement, à partir de manuscrits divers dont les plus anciens remontent au $\mathrm{XIII}^{\mathrm{e}}$ siècle et font partie des témoins d'une transmission des savoirs dits «magiques» de l'Antiquité au

27. Michael Houseman, «Comment comprendre l'esthétique affectée des cérémonies New Age et néopaïennes ? », La force des objets - Matières à expérience, dir. Jean-Pierre Albert, Anouk Cohen, Agneszka Kedzierska-Manzon, Damien Mottier, Archives des sciences sociales des religions, t. 174, 2016, p. 213-237.

28. Sur l'image du « bricoleur », voir Claude Lévi-Strauss, La pensée sauvage, Paris, Plon, 1962, p. 30-36. 
Moyen Âge, en particulier dans le monde byzantin ${ }^{29}$. Il s'agit d'une transmission dynamique, d'une composition vivante qui résulte en une sorte d'agrégat de données dont il est difficile de saisir les sources d'emprunt et la diachronie. Dans la veine des grimoires ou des physica (comme on commence à les appeler à la fin de l'Antiquité), le livre propose des séries d'amulettes et d'autres recettes organisées par regroupement ou compositions de plantes, pierres et animaux dont les noms commencent par la même lettre - le tout classé par ordre alphabétique. Cette opération de systématisation s'accompagne, au besoin, de la production de mots pour nommer les éléments qui doivent entrer sous une même rubrique de manière à n'avoir que des noms commençant par la même lettre. C'est le cas par exemple à la lettre delta, qui regroupe la plante drakontios ou serpentaire, le dendrokolaptês («piquearbre ») ou pic-vert, le drakôn marin qui est la vive, et le dendritês, une pierre « arborescente » comparée au corail et dite venir d'Inde. Les noms croisent deux familles lexicales, celle du serpent ou dragon (drakôn) et celle de l'arbre (dendron). Les Cyranides emploient dendrokolaptês plutôt que druokolaptês ( « pique-chêne ») sans doute pour favoriser la consonance, et nous exposent que :

Si quelqu'un bouche avec de la terre ou une pierre le nid du dendrokolaptês, celui-ci apporte une herbe dont il a connaissance et le réouvre en l'approchant. S'il est bloqué par de la boue, la boue tombe aussi, et si c'est par une pierre, la pierre s'éjecte ; si c'est une planche et des clous, ils tombent, expulsés; si c'est une plaque de fer et des clous ils éclatent. Car en effleurant seulement avec cette herbe, il ouvre absolument tout sur-le-champ et reprend son nid. Si quelqu'un découvre cette herbe, il fera beaucoup de choses dont on ne peut parler, car il pourra faire ${ }^{30}$, grâce à sa puissance naturelle, ce qu'aucun homme n'accomplirait. ${ }^{31}$

Les Cyranides reprennent un savoir attesté déjà au I ${ }^{\mathrm{er}}$ siècle sur le pic-vert, connaisseur d'une plante capable de débloquer le trou

29. Jeffrey Spier, «A Revival of Antique Magical Practice in Tenth-Century Constantinople », Magic and the Classical Tradition, éd. Charles Burnett, William F. Ryan, Londres/Turin, The Warburg Institute/Nino Aragno Editore, 2006, p. 29-36 ; «An Antique Magical Book Used for Making Sixth-Century Byzantine Amulets ?», La transmission des savoirs magiques, éd. Véronique Dasen, JeanMichel Spieser, Florence, Sismel - Edizioni del Galluzzo, 2014, p. 43-66.

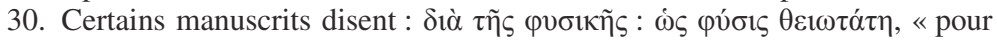
des raisons naturelles : puisque la nature est très divine ».

31. Cyranides, I, 4, 36-44. 
qu'il a creusé dans l'arbre en cas d'obstruction ${ }^{32}$. Ce n'est pas à proprement parler une tradition rituelle, mais un savoir naturaliste. Néanmoins, la technique s'empare du savoir pour construire une amulette, un objet puissant :

Si quelqu'un grave sur la pierre dendritês le dendrokolaptês avec sous ses pieds le drakôn de mer et la porte, après avoir enfermé dessous l'herbe qui a été trouvée par le dendrokolaptês, cette personne ouvrira avec cela toutes les portes, déverrouillera les liens et les serrures, les bêtes sauvages lui seront soumises et apprivoisées, il sera plaisant et agréable à écouter pour tous les dieux et les hommes. Et il réussira en tout ce qu'il souhaite et surpassera ce qu'il voudra. ${ }^{33}$

La «recette» ne propose ni plus ni moins qu'un moyen «naturel » d'acquérir pour soi le miracle à travers lequel, dans d'autres documents et dans des représentations religieuses différentes ("paganisme» et «christianisme»), c'est l'intervention du divin qui débarrasse certains privilégiés de leurs entraves et les tire de prison $^{34}$. La technique est effectivement innovante, mais elle s'inscrit dans une tradition merveilleuse, qui plus est interculturelle. Le pouvoir des plantes, pierres et animaux ne peut être véritablement expliqué par les humains qui les constatent et les relatent: c'est une puissance naturelle

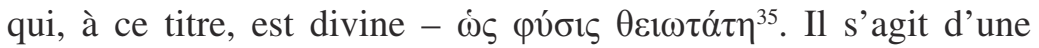
première légitimation d'un savoir technique qui innove à partir de connaissances précédentes. D'autres moyens peuvent être sollicités pour valider le savoir: pour l'amulette ici retenue, il a été jugé nécessaire, à un moment de la composition du texte, de citer un hymne attribué à une autorité fondatrice : "On dit aussi un hymne à Hermès Trismégiste qui est le guide de toute sagesse,

32. Pline l'Ancien, Histoire naturelle, X, 20; XXV, 5 (avec référence à Théophraste) ; Élien, Personnalité des animaux, I, 45.

33. Cyranides, I, 4, 45-54.

34. Les bacchantes par Dionysos (Euripide, Les Bacchantes, 443-448); l'apôtre Pierre par l'ange du Seigneur (Actes des apôtres, V, 18-20; XII, 1), ou Paul et Silas (Actes des apôtres, 16, 22-40); voir Jean-Marie Pailler, «Pierre et Paul aux liens. Entre réel et symbolique, les métamorphoses d'une double expérience (30-50 apr. J.-C.) », Ekklèsia. Approches croisées d'histoire politique et religieuse. Mélanges offerts à Marie-Françoise Baslez, éd. Corinne Bonnet, Françoise Briquel-Chatonnet, Pallas, t. 104, 2017, p. 207-224. C'est ce que peut accomplir le dieu paredros invoqué grâce au rituel de Pnouthis, hiérogrammate, $P G M$ I, 101 : «il délie de leurs liens ceux qui sont enchaînés en garde-à-vue, il

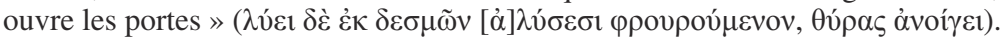

35. Voir supra n. 30. 
le conducteur des paroles, le pourvoyeur très savant de tout art et le plus merveilleux des astres $»^{36}$. L'hymne vante, justement, les pouvoirs de l'amulette et même bien plus :

En portant cela tu apprendras tout ce qui concerne les dieux, tu ouvriras tous les verrous et délieras toutes les entraves, tu apprivoiseras absolument toutes les bêtes, tous les oiseaux viendront à toi, tu apprivoiseras les bêtes de la mer comme un dieu. Tu arrêteras le flot terrible et sauvage, tous les démons te fuiront toujours. Toi seul paraîtras bon à tous les hommes. ${ }^{37}$

Ce qui est ici le centre de l'attention, ce sont les pouvoirs merveilleux qui doivent être validés par une figure de tradition et d'autorité, Hermès Trismégiste, et une forme de discours lui-même traditionnel et codifié pour qui veut plaire aux dieux ${ }^{38}$, l'hymne - dont les vers hélas ont été brouillés dans la tradition manuscrite. La composition de l'amulette et la composition du texte sont un exemple de bricolage qui construit un savoir reposant à la fois sur les vertus naturelles des plantes et des pierres, sur l'efficience de certaines images et sur l'autorité de référents traditionnels qui sont convoqués pour ancrer l'innovation dans un passé lointain. Ce faisant, le savoir est une tradition qui s'invente en continu et construit perpétuellement l'autorité dont il tire une légitimation et une garantie d'efficacité.

Les experts rituels dont nous classons les productions comme « magiques » sont tout à la fois passeurs et faiseurs de traditions, naturalistes et religieuses. Il est nécessaire, pour comprendre leur activité, de leur reconnaitre cette triangulation entre tradition, innovation et autorité : la double opération de transmission et d'invention garde en ligne de mire la question de la validité du savoir, qui est un enjeu majeur pour le crédit de l'expert. Cette autorité confère une force exécutoire aux pratiques, valide la tradition même que le praticien ou le rédacteur de textes « magiques » reçoit, voire invente ; elle peut, également, répondre à l'autorité institutionnalisée à laquelle le praticien fait face et qui l'identifie, précisément, comme «magicien». En adaptant les

36. Cyranides, I, 4, 52-54. L'édition de Dimitri Kaimakis est ici, comme en bien d'autres endroits, source de possibles malentendus. L'éditeur fournit un texte établi d'après les manuscrits, eux-mêmes très hétéroclites, et une reconstitution versifiée de l'hymne.

37. Cyranides, I, 4, 58-63.

38. I.Didyma, 217, $8:$ : Tous les chants me réjouissent ». 
pratiques hors d'une structure collective, l'expert rituel ne peut se passer de fonder son savoir sur une autorité ou des modèles passés qui, indépendamment de toute catégorisation, assurent à sa propre expertise une place dans la compétition pour le pouvoir rituel. Quel que soit le degré de marginalité que l'on tente de reconnaitre à ces experts rituels, et précisément parce qu'ils sont relégués dans une «marginalité », ils n'en montrent pas moins la tension inhérente dans les affaires religieuses entre tradition et innovation, puisque leur efficacité construit aussi leur autorité. Comme le pic-vert, l'expert rituel que nous appelons bien souvent «magicien» sait se munir des outils pour ouvrir la tradition dont il hérite ou qu'il contribue à bâtir.

thomas.galoppin@univ-tlse2.fr 